\title{
TdT expression in acute myeloid leukemia with minimal differentiation is associated with distinctive clinicopathological features and better overall survival following stem cell transplantation
}

\author{
Keyur P Patel ${ }^{1}$, Faisal A Khokhar ${ }^{1}$, Tariq Muzzafar ${ }^{1}$, M James You ${ }^{1}$, Carlos E Bueso-Ramos ${ }^{1}$, \\ Farhad Ravandi ${ }^{2}$, Sherrie Pierce ${ }^{2}$ and L Jeffrey Medeiros ${ }^{1}$ \\ ${ }^{1}$ Department of Hematopathology, The University of Texas MD Anderson Cancer Center, Houston, TX, USA \\ and ${ }^{2}$ Department of Leukemia, The University of Texas MD Anderson Cancer Center, Houston, TX, USA
}

The diagnostic criteria for acute myeloid leukemia (AML), not otherwise specified, with minimal differentiation (AML-M0, French-American-British classification), have been refined in the 2008 World Health Organization (WHO) classification. Terminal deoxynucleotidyl transferase (TdT) expression in AML-MO has been proposed by others as a surrogate for RUNX1 (runt-related transcription factor 1) mutations, a mutation associated with distinct gene expression profiles in AML-MO. In this study, we investigated the significance of TdT expression in AML-MO cases defined using the 2008 WHO classification criteria. Demographic, laboratory and clinical information were obtained from the hospital medical records. Statistical analysis was performed using Student's $t$-test, log-rank test and Fisher's exact test. The study group included 30 AML-MO patients (male:female $=19: 11$; median age: 60 years). In all, 10 cases of AML-M0 were positive for $\operatorname{TdT}(+)$ and 20 cases were negative for $\operatorname{TdT}(-)$. Patients with TdT + AML-M0 had higher peripheral blood and bone marrow blast counts compared to patients with TdT - AML-MO $(P=0.01)$. TdT expression in AML-M0 was not associated with a distinct immunophenotype. Monoclonal IgH and TCR gene rearrangements were frequent, but independent of TdT expression in AML-MO. TdT expression in AML-MO correlated with trisomy 13 and inversely correlated with aberrations of chromosomes 5 and 17. Among six patients with AML-MO who received a stem cell transplant, overall survival was significantly longer for the three TdT + patients compared with the three TdT - patients $(P=0.03)$. In the TdT + AML-M0 subgroup, the three patients with stem cell transplant had better overall survival compared with five patients who did not receive stem cell transplant $(P=0.01)$. We conclude that AML-M0, as currently defined in the 2008 WHO classification, can be divided into two groups based on TdT expression. Although there is a need to assess a greater number of patients, our results suggest that TdT positivity in AML-MO identifies a subset of patients with a better prognosis after stem cell transplant.

Modern Pathology (2013) 26, 195-203; doi:10.1038/modpathol.2012.142; published online 31 August 2012

Keywords: acute myeloid leukemia; AML-M0; minimal differentiation; RUNX mutations; TdT

Acute myeloid leukemia (AML), not otherwise specified, with minimal differentiation, is a type of AML without evidence of myeloid differentiation by

Correspondence: Dr LJ Medeiros, MD, Department of Hematopathology, Unit 72, The University of Texas MD Anderson Cancer Center, 1515 Holcombe Boulevard, Houston, TX 77030, USA.

E-mail: ljmedeiros@mdanderson.org

Received 25 February 2012; revised 7 May 2012; accepted 14 May 2012; published online 31 August 2012 light microscopy morphology and cytochemistry. ${ }^{1}$ This category corresponds, in part, to AML-M0 in the French-American-British (FAB) classification scheme, ${ }^{2}$ and based on convenience, the AML-M0 terminology is used in this study. Patients with AML-M0 represent approximately $5 \%$ of all patients with AML, with infants or older adults most often affected, and patients usually have a poor prognosis with low remission rate. ${ }^{1-5}$ The blasts of AML-M0 are usually medium sized, but they can be small, 
resembling lymphoblasts. By definition, myeloperoxidase is present in $<3 \%$ of AML-Mo blasts by cytochemistry; however, the blasts have immunophenotypic evidence of myeloid lineage shown by expression of early myeloid antigens such as CD13, CD33 or CD117. Mature myeloid/monocytic markers are usually not expressed. Nuclear terminal deoxynucleotidyl transferase (TdT) expression is reported to be positive in approximately $50 \%$ of AML-M0 cases. ${ }^{1-6}$ No specific chromosomal abnormalities have been associated with AML-M0. ${ }^{1}$

Although the category of AML, not otherwise specified, with minimal differentiation has been recognized by the World Health Organization (WHO) classification since 2001, the criteria for diagnosis have been refined in the more recent edition of the WHO system. ${ }^{1}$ In particular, in older literature a subset of AML-M0 cases were reported to have a history of a myelodysplastic syndrome, simultaneous evidence of myelodysplasia or complex and unbalanced cytogenetic changes. ${ }^{3,7}$ These cases are now likely to be classified as AML with myelodysplasiarelated changes in the current WHO system as shown in Figure $1 .^{1}$ As a result, the frequency of AML with minimal differentiation is reduced and the category has become more 'exclusive'. Thus, older literature describing the clinicopathological and cytogenetic features of AML-M0 patients does not match with cases currently classified as AMLMo, and we are not aware of any clinicopathological studies describing AML-MO using the new WHO criteria.

To date, no single genetic mechanism explaining leukemogenesis in cases of AML-M0 has been identified. However, RUNX1 (runt-related transcription factor 1), a transcription factor involved in hematopoietic differentiation, has been shown to be mutated frequently in AML-M0, and is associated with poorer clinical outcome. ${ }^{8-10}$ Mutations in RUNX1 are clustered within, but are not restricted to, the Runt homology domain, and therefore comprehensive mutational analysis requires sequencing of at least eight exons. ${ }^{8,9,11}$ Clinical testing for RUNX1 mutations is therefore not routinely performed. Recent genome-wide analyses has shown that TdT expression is upregulated in AML-M0 with RUNX1 mutation. ${ }^{10,12}$ Three TdT probe sets were among the top four in an eight probe set classifier used to identify AML-M0 with RUNX1 mutation. ${ }^{10}$ As TdT expression is routinely tested by flow cytometry for all new acute leukemias, TdT may be useful as a convenient surrogate for RUNX1 mutation.

The aims of this single institution study were to review cases of AML with minimal differentiation as defined using the current WHO classification criteria, and to subdivide this group according to TdT expression. To the best of our knowledge, this study provides the first description of the clinicopathological characteristics of AML-M0 cases defined with the current WHO criteria. This study also shows that the AML-M0 category remains heterogeneous and that subdivision according to TdT expression identifies two distinct subsets of patients.

\section{Methods}

\section{Patient Selection}

The study was approved by the Institutional Review Board of MD Anderson Cancer Center. An informed consent was obtained from all patients included in the study. A total of 30 patients with de novo AML, not otherwise specified, with minimal differentiation were identified in the files of our department for the time interval 1 January 2002 to 31 December 2008. We excluded from the study group any patients with history or concurrent evidence of myelodysplasia, myeloproliferative neoplasm or therapy-related myeloid neoplasms. Patients who received chemotherapy previously were also excluded. For the final study group, medical records were reviewed with regard to therapy, including stem cell transplant, as well as response to therapy and survival.

\section{Cytochemistry and Immunophenotyping}

Flow cytometric immunophenotyping was performed on bone marrow aspirates in all patients and was analyzed using multicolor analysis and a FACScan instrument (BD Biosciences, San Jose, CA, USA) as described previously. ${ }^{13}$ The panel of antibodies included: CD2, surface CD3, cytoplasmic CD3, CD4, CD5, CD7, CD8, CD10, CD13, CD19, CD20, cytoplasmic CD22, CD33, CD34, CD38, CD45, CD52, CD56, CD64, CD123 and TdT.

\section{Cytogenetics and Molecular Studies}

All cases were analyzed by conventional cytogenetics analysis as described previously. ${ }^{13}$ Molecular testing for mutations in RAS (KRAS and NRAS) and FLT3 was performed as described previously. ${ }^{14,15}$ PCR-based testing was performed to analyze the immunoglobulin heavy chain gene (IGH), T-cell receptor $\beta$ gene (TCRB) and T-cell receptor $\gamma$ gene $(T C R G)$ as described previously. ${ }^{16,17}$

\section{Statistical Analysis}

Statistical analysis was performed using Student's two-tailed $t$-test, log-rank test, and Fisher's twotailed exact test as applicable. 


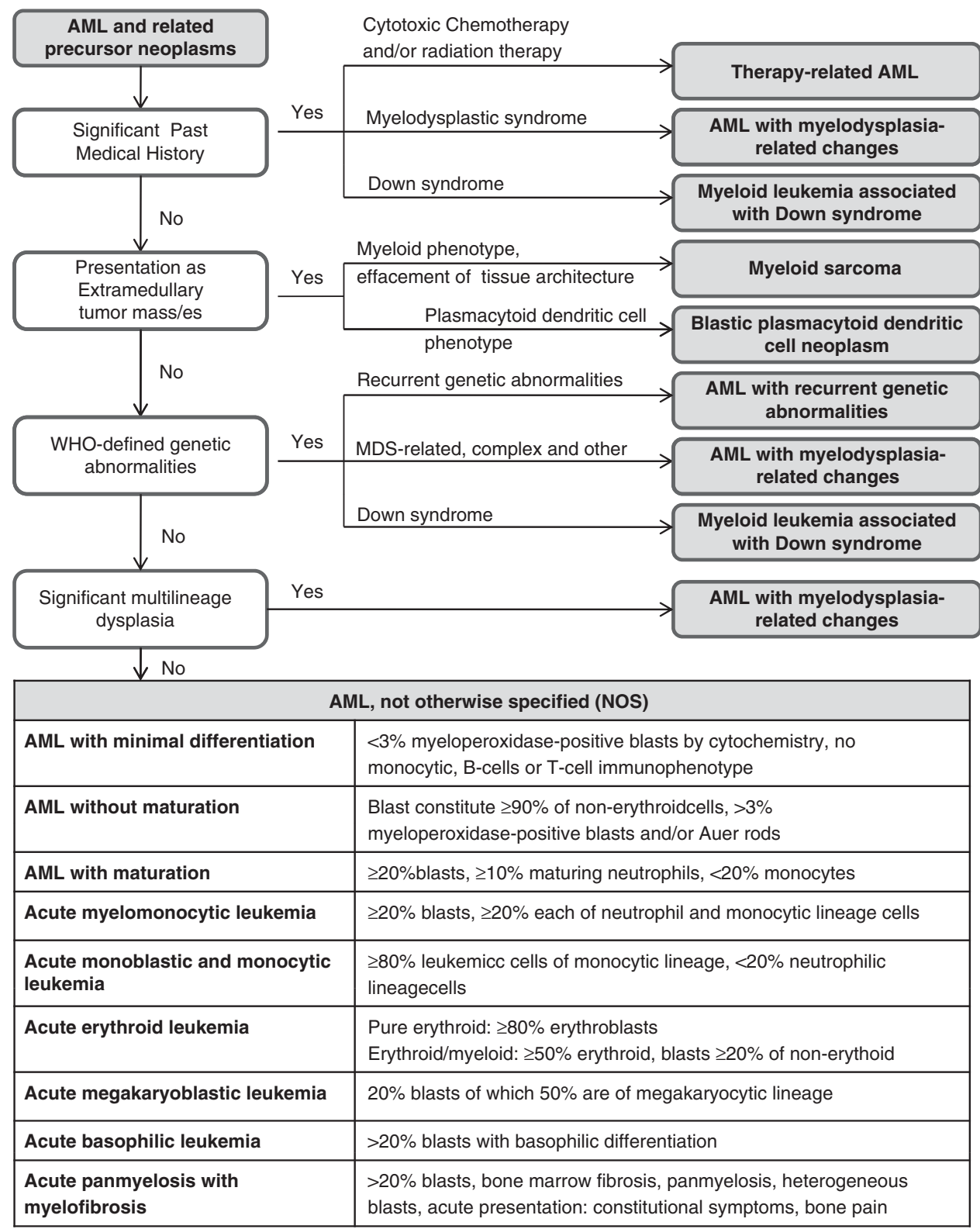

Figure 1 Algorithmic approach for subclassification of acute myeloid leukemia using the 2008 World Health Organization (WHO) classification.

\section{Results}

\section{TdT Expression in AML-Mo is Associated with Higher Blast Counts}

The study group (Table 1) included 19 male and 11 female subjects with a median age of 60 years (range, 16-87). A complete blood count at diagnosis showed cytopenias. The median white blood cell (WBC) count was $2.2 \times 10^{9} / \mathrm{l}$ (range, 0.3-226; reference range, 4-11), hemoglobin 9.7 g/dl (range, 5.6-13.3; reference range, 14-18) and platelet count $46 \times 10^{9} / 1$ (range, 11-272; reference range, 140-440). The median peripheral blood blast count was 52\% (range, 0-99). The median lactate dehydrogenase
(LDH) was 635 IU/l (range, 334-15 544; normal range, 313-618). In all, 16 patients had an elevated serum LDH. Other chemistry studies were unremarkable. Bone marrow aspirate smears showed numerous blasts, with a median of 78\% (range, 20-98).

Using a cutoff of $25 \%$ blasts positive for TdT, 10 $(33.3 \%)$ cases were $\mathrm{TdT}+$ and 20 cases were $\mathrm{TdT}-$. Peripheral blood (median, 77 versus 14; $P=0.04$ ) and bone marrow (median, 84 versus $71 ; P=0.01$ ) blast percentages were significantly higher in patients with TdT + AML-M0 versus TdT - AML-M0. There were no other statistically significant differences in clinical findings between the two subgroups, although the TdT + AML-M0 group 
Table 1 Demographics and Clinical Presentation of AML-M0 patients

\begin{tabular}{|c|c|c|c|c|}
\hline Characteristic & $\begin{array}{c}\text { Total } \\
(\mathrm{N}=30)\end{array}$ & $\begin{array}{c}T d T+ \\
(\mathrm{N}=10)\end{array}$ & $\begin{array}{c}T d T- \\
(\mathrm{N}=20)\end{array}$ & $\begin{array}{c}\mathrm{P}- \\
\text { value }\end{array}$ \\
\hline Gender & & & & $0.7^{\mathrm{a}}$ \\
\hline $\mathrm{M}: \mathrm{F}$ & $19: 11$ & $7: 3$ & $12: 8$ & \\
\hline Age (years) & & & & $0.37^{b}$ \\
\hline Average & 57.8 & 62.6 & 55.3 & \\
\hline Median & 60 & 65.5 & 57 & \\
\hline Range & $16-87$ & $17-83$ & $16-87$ & \\
\hline $\mathrm{WBC}(\mathrm{k} / \mu \mathrm{l})$ & & & & $0.23^{\mathrm{b}}$ \\
\hline Average & 25.5 & 45.9 & 15.3 & \\
\hline Median & 2.2 & 5.05 & 2.05 & \\
\hline Range & $0.3-226$ & $1-226$ & $0.3-178$ & \\
\hline $\mathrm{Hgb}(\mathrm{g} / \mathrm{dl})$ & & & & $0.8^{\mathrm{b}}$ \\
\hline Average & 9.7 & 9.8 & 9.6 & \\
\hline Median & 9.7 & 9.5 & 9.8 & \\
\hline Range & $5.6-13.3$ & $7.6-12.6$ & $5.6-13.3$ & \\
\hline Platelet $(\mathrm{k} / \mu \mathrm{l})$ & & & & $0.9^{b}$ \\
\hline Average & 75 & 77 & 74 & \\
\hline Median & 46 & 60 & 45 & \\
\hline Range & $11-272$ & $23-272$ & $11-229$ & \\
\hline $\begin{array}{l}\text { Peripheral blood } \\
\text { blast (\%) }\end{array}$ & & & & $0.04^{\mathrm{b}}$ \\
\hline Average & 44 & 68 & 34 & \\
\hline Median & 52 & 77 & 14 & \\
\hline Range & 0-99 & 0-99 & $0-92$ & \\
\hline $\begin{array}{l}\text { Bone marrow } \\
\text { blast (\%) }\end{array}$ & & & & $0.01^{\mathrm{b}}$ \\
\hline Average & 70 & 81 & 65 & \\
\hline Median & 78 & 84 & 71 & \\
\hline Range & $20-98$ & $58-98$ & $20-96$ & \\
\hline LDH (U/l) & & & & $0.26^{\mathrm{b}}$ \\
\hline Average & 1358 & 759 & 1657 & \\
\hline Median & 635 & 608 & 673 & \\
\hline Range & 334-15 544 & 349-1828 & 334-15 544 & \\
\hline $\mathrm{LDH}$ & & & & $0.71^{\mathrm{a}}$ \\
\hline In normal range & 14 & 4 & 10 & \\
\hline Increased & 16 & 6 & 10 & \\
\hline
\end{tabular}

${ }^{\mathrm{a}}$ Fisher's two-tailed exact test.

b'Student's two-tailed $t$-test.

appeared to have a higher male:female ratio, median age, WBC count and platelet count, and the TdT AML-M0 group appeared to have higher serum LDH levels (Table 1).

TdT Expression in AML-Mo is not Associated with a Distinct Morphology or Immunophenotype

In the study group, no correlation was observed between $\mathrm{TdT}+$ and $\mathrm{TdT}-$ cases and blast or bone marrow morphology. By definition, all cases of AML-M0 were negative for myeloperoxidase expression $(<3 \%$ positive blasts) by cytochemistry and flow cytometry. Of $30(43 \%)$ cases, 13 showed expression of a $\mathrm{T}$ - or B-cell-associated antigen
(CD2, CD5, CD7 or CD19), but these cases did not meet the criteria for mixed phenotype acute leukemia, NOS, as specified in the current WHO classification (Table 2). No significant differences in the expression of T- or B-cell antigens were observed between TdT + and TdT - AML-M0. No significant differences were observed in the expression of CD13, CD33, CD34, CD56, and CD117 between TdT + and TdT - AML-M0. No significant association was observed between the percentage of blasts expressing $\mathrm{TdT}$ and expression of lymphoid markers.

TdT Expression in AML-Mo Correlates with Trisomy 13 and Inversely Correlates with Aberrations of Chromosomes 5 and 17

Conventional karyotyping of bone marrow aspirates from 30 AML-M0 cases showed a diploid karyotype in $8(27 \%)$, a non-complex $(<3$ chromosomal abnormalities) karyotype in $7(23 \%)$ and a complex karyotype ( $\geq 3$ chromosomal abnormalities) in 15 $(50 \%)$. On the basis of the number and type of chromosomal aberrations, 16 (53\%) patients had intermediate-risk and the remaining patients had poor-risk cytogenetics. No significant differences in the cytogenetic risk groups were observed between patients with TdT + and TdT - AML-M0 (Table 2).

The most frequent chromosomal abnormalities in AML-M0 involved chromosomes 5 (8/30, 27\%), 7 (8/ $30,27 \%), 11(7 / 30,23 \%), 17$ (7/30, 23\%), 13 (6/30, $20 \%), 8(6 / 30,20 \%)$ and $21(4 / 30,13 \%)$ (Table 2). Chromosome 13 gains, two with trisomy 13 and one with tetrasomy 13 , were only identified in patients with TdT + AML-M0 (3/10; 30\%; $P=0.03)$. All TdT - AML-M0 cases with abnormalities involving chromosome 13 showed loss or deletion (3/20; 15\%; $P=0.5)$. Aberrations of chromosome $5(8 / 20 ; 40 \%)$ or chromosome 17 (7/20;35\%) were identified only in patients with TdT - AML-M0 ( $P=0.03$ for each). There were no other significant differences in the frequency or nature of chromosomal aberrations between the TdT + and TdT - AML-M0 groups.

\section{Monoclonal IGH and TCR Gene Rearrangements are Frequent in AML-M0 and are Independent of TdT Expression}

Previous studies have shown frequent monoclonal antigen receptor gene rearrangements in AML-M0 cases. ${ }^{18}$ In this study, based on the analysis of bone marrow aspirate samples, monoclonal antigen receptor gene rearrangements were observed in 12 of $23(52 \%)$ cases tested. T-cell receptor $\gamma$ or T-cell receptor $\beta$ or both gene rearrangements were most frequent in 11 of $23(48 \%)$ cases. Monoclonal IGH gene rearrangements were identified in 4 of 18 $(22 \%)$ cases tested. No significant differences were observed in the frequency of gene rearrangements in $\mathrm{TdT}+$ versus $\mathrm{TdT}-$ AML-M0 cases (Table 2). 
Table 2 Selected Molecular, Cytogenetic and Immunophenotypic Features of AML-Mo Cases

\begin{tabular}{|c|c|c|c|c|}
\hline Characteristic & Total $(\mathrm{N}=30)$ & $T d T+(\mathrm{N}=10)$ & $T d T-(\mathrm{N}=20)$ & P-value (Fisher's two-tailed exact test) \\
\hline \multicolumn{5}{|l|}{ Molecular } \\
\hline FLT3 & & & & \multirow[t]{3}{*}{0.34} \\
\hline Mutant & $5 / 28(18 \%)$ & $3 / 10(30 \%)$ & $2 / 18(11 \%)$ & \\
\hline WT & $23 / 28(82 \%)$ & $7 / 10(10 \%)$ & $16 / 18(89 \%)$ & \\
\hline$R A S$ & & & & \multirow[t]{3}{*}{0.53} \\
\hline Mutant & $3 / 25(12 \%)$ & $0 / 7$ & $3 / 18(17 \%)$ & \\
\hline WT & $22 / 25(88 \%)$ & 7/7 (100\%) & 15/18 (83\%) & \\
\hline$I G H$ & & & & \multirow[t]{3}{*}{0.57} \\
\hline Clonal & $4 / 18(22 \%)$ & $3 / 9(33 \%)$ & $1 / 9(11 \%)$ & \\
\hline Non-clonal & $14 / 18(78 \%)$ & $6 / 9(67 \%)$ & $8 / 9(89 \%)$ & \\
\hline TCR- $\beta / \gamma$ & & & & \multirow[t]{3}{*}{0.41} \\
\hline Clonal & $11 / 23(48 \%)$ & $6 / 10(60 \%)$ & $5 / 13(38 \%)$ & \\
\hline Non-clonal & $12 / 23(52 \%)$ & $4 / 10(40 \%)$ & $8 / 13(62 \%)$ & \\
\hline IGH/TCRB/TCRG & & & & \multirow[t]{3}{*}{0.42} \\
\hline Clonal & $12 / 23(52 \%)$ & $6 / 10(60 \%)$ & $6 / 13(46 \%)$ & \\
\hline Non-clonal & $11 / 23(48 \%)$ & $4 / 10(40 \%)$ & $7 / 13(54 \%)$ & \\
\hline \multicolumn{5}{|l|}{ Cytogenetics } \\
\hline \multicolumn{5}{|l|}{ Karyotype } \\
\hline Diploid & $8 / 30(27 \%)$ & $3 / 10(30 \%)$ & $5 / 20(25 \%)$ & 1.00 \\
\hline Simple & $7 / 30(23 \%)$ & $3 / 10(30 \%)$ & $4 / 20(20 \%)$ & 0.41 \\
\hline Complex & $15 / 30(50 \%)$ & $4 / 10(40 \%)$ & $11 / 20(55 \%)$ & 0.46 \\
\hline \multicolumn{5}{|l|}{ Risk category } \\
\hline Favorable & 0 & 0 & 0 & \\
\hline Intermediate & $15 / 30(50 \%)$ & $6 / 10(60 \%)$ & 9/20 (45\%) & 0.47 \\
\hline Poor & $15 / 30(50 \%)$ & $4 / 10(40 \%)$ & $11 / 20(55 \%)$ & 0.47 \\
\hline \multicolumn{5}{|l|}{ Chromosomes } \\
\hline 5 & $8 / 30(27 \%)$ & $0 / 10$ & $8 / 20(40 \%)$ & 0.03 \\
\hline 7 & $8 / 30(27 \%)$ & $1 / 10(10 \%)$ & $7 / 20(35 \%)$ & 0.20 \\
\hline 8 & $6 / 30(20 \%)$ & $2 / 10(20 \%)$ & $4 / 20(20 \%)$ & 1.00 \\
\hline 11 & $7 / 30(23 \%)$ & $3 / 10(30 \%)$ & $4 / 20(20 \%)$ & 0.67 \\
\hline+13 & $3 / 30(10 \%)$ & $3 / 10(30 \%)$ & $0 / 20$ & 0.03 \\
\hline-13 & $3 / 30(10 \%)$ & $0 / 10$ & $3 / 20(15 \%)$ & 0.5 \\
\hline 17 & $7 / 30(23 \%)$ & $0 / 10$ & $7 / 20(35 \%)$ & 0.03 \\
\hline 21 & $4 / 30(13 \%)$ & $1 / 10(10 \%)$ & $3 / 20(15 \%)$ & 1.00 \\
\hline \multicolumn{5}{|l|}{ Immunophenotype } \\
\hline CD2 & $2 / 26(8 \%)$ & $1 / 10(10 \%)$ & $1 / 16(6 \%)$ & 1.00 \\
\hline CD3 & $0 / 30$ & $0 / 10$ & $0 / 20$ & \\
\hline CD5 & 4/27 (15\%) & $0 / 10$ & 4/17 (24\%) & 0.13 \\
\hline CD7 & 9/27 (33\%) & $3 / 10(30 \%)$ & $6 / 17(35 \%)$ & 1.00 \\
\hline CD19 & 4/29 (14\%) & $2 / 10(20 \%)$ & 2/19 (11\%) & 0.61 \\
\hline Any T or B cell & $13 / 30(43 \%)$ & $4 / 10(40 \%)$ & $9 / 20(45 \%)$ & 0.72 \\
\hline
\end{tabular}

${ }^{\mathrm{a}}$ No significant difference in the expression of CD34, CD117, HLA-DR, CD13, CD33 and CD56.

FLT3 gene mutations were identified in 5 of 28 $(18 \%)$ and $R A S$ (KRAS and NRAS) gene mutations in 3 of $25(12 \%)$ AML-M0 cases tested. FLT3 mutations occurred in both TdT + and TdT - AML-M0 patients with similar frequency $(P=0.34)$. KRAS/ NRAS mutations were limited to patients with TdT AML-M0 (3/18, 17\%; $P=0.53)$ (Table 2).

\section{Patients with TdT + AML-Mo Show Better Overall Survival Following Stem Cell Transplant Compared with Patients with TdT + AML-Mo Without Transplant or Patients with TdT - AML-Mo with Transplant}

Analysis of available clinical outcome in response to the induction chemotherapy (Table 3) in 26 AMLMo patients showed complete remission (CR) in 13 (50\%), CR with incomplete bone marrow recovery (CRi) in $1(4 \%)$, partial response (PR) in $5(19 \%)$ and refractory AML in 7 (\%) (Table 1). Overall, 17 of 26 $(65 \%)$ patients achieved CR and 9 (35\%) patients did not. Average and median overall survival of AML-M0 patients were 23.5 and 10.6 months, respectively (range, 2.4-96.6). In 24 patients with available survival information, 1- and 5-year survival rates were 11 of $24(46 \%)$ and 2 of $24(8 \%)$, respectively.

Patients with TdT + AML-M0 appeared to show a slightly better 5 -year survival $(2 / 9,22 \%)$ compared with patients with TdT - AML-M0 (0/15); however, this difference did not reach statistical significance $(P=0.13)$. There was no significant difference in induction responses (CR, CRi, PR, refractory), achievement of CR, overall survival and 1-year survival between patients with $\mathrm{TdT}+$ versus $\mathrm{TdT}-$ AML-M0 (Table 2).

Available clinical information showed that 6 of 26 patients received stem cell transplant, including 3 of 
Table 3 Clinical Outcome of AML-M0

\begin{tabular}{|c|c|c|c|c|}
\hline Characteristic & $\begin{array}{c}\text { Total } \\
(\mathrm{N}=30)\end{array}$ & $\begin{array}{c}T d T+ \\
(\mathrm{N}=10)\end{array}$ & $\begin{array}{c}T d T- \\
(\mathrm{N}=20)\end{array}$ & $\begin{array}{c}\mathrm{P}- \\
\text { value }\end{array}$ \\
\hline \multicolumn{5}{|l|}{ Induction response } \\
\hline CR & 13 & 4 & 9 & $1.00^{\mathrm{a}}$ \\
\hline CRi & 1 & 0 & 1 & $1.00^{\mathrm{a}}$ \\
\hline $\mathrm{PR}$ & 5 & 2 & 3 & $1.00^{\mathrm{a}}$ \\
\hline Refractory & 7 & 2 & 5 & $1.00^{\mathrm{a}}$ \\
\hline Not available & 5 & 2 & 2 & \\
\hline Achieved CR & & & & $0.67^{\mathrm{a}}$ \\
\hline Yes & 17 & 6 & 11 & \\
\hline No & 9 & 2 & 7 & \\
\hline Not available & 5 & 2 & 2 & \\
\hline Current status & & & & $0.03^{\mathrm{a}}$ \\
\hline Alive & 3 & 3 & 0 & \\
\hline Deceased & 25 & 6 & 19 & \\
\hline NA & 3 & 1 & 1 & \\
\hline $\begin{array}{l}\text { Overall survival } \\
\text { (months) }\end{array}$ & $N=24$ & $N=9$ & $N=15$ & $0.2^{\mathrm{b}}$ \\
\hline Average & 23.5 & 34.7 & 16.7 & \\
\hline Median & 10.6 & 20 & 8.4 & \\
\hline Range & $2.4-96.6$ & $3.3-96.6$ & $2.4-57.9$ & \\
\hline 1-Year survival rate & $\begin{array}{l}11 / 24 \\
(46 \%)\end{array}$ & $5 / 9(56 \%)$ & $6 / 15(40 \%)$ & $0.68^{\mathrm{b}}$ \\
\hline 5-Year survival rate & $2 / 24(8 \%)$ & $2 / 9(22 \%)$ & $0 / 15$ & $0.13^{\mathrm{b}}$ \\
\hline
\end{tabular}

CR, complete remission; CRi, complete remission incomplete marrow recovery; PR, partial response.

${ }^{\text {a}}$ Fisher's two-tailed exact test.

bstudent's two-tailed $t$-test.

$8(37.5 \%) \mathrm{TdT}+$ versus 3 of $18(16.7 \%) \mathrm{TdT}-$ AML-M0 patients. Overall survival was significantly longer for the three patients with TdT + AML-M0 (43.2, 91.3 and 97.4 months; average: 76.3 months) compared with the three TdT - AML-M0 patients (4.7, 10.3 and 11.7 months; average: 8.9 months) $(P=0.03$, log-rank test). Similar beneficial outcome was noted within the TdT + AML-M0 group. The three TdT + AML-M0 patients who received stem cell transplant had better overall survival (43.2, 91.3 and 97.4 months; average: 76.3 months) compared with five TdT + AML-M0 patients who did not receive stem cell transplant (5.1, 5.1, 9.5, 20 and 40.2 months; average: 16 months $)(P=0.01$, log-rank test). The overall survival is likely to improve for the TdT + AML-M0 group because all three patients receiving stem cell transplant are still alive. There was no significant difference in the cytogenetic data, response to induction therapy or achievement of CR between the groups, suggesting that TdT expression can be used as an indicator of beneficial outcome in patients treated with stem cell transplant (data not shown).

Characteristics of TdT + AML-Mo in this Study Differ from Those Reported for AML-M0 with RUNX1 Mutation

Recent studies showed a high degree of correlation between RUNX1 mutation and TdT expression in
AML-M0. ${ }^{10,12}$ We compared the characteristics of TdT + AML-M0 in our study with other reports of

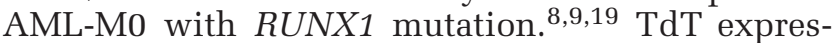
sion in this series, $10 / 30(33 \%)$, is comparable to the reported frequency of $R U N X 1$ mutations, $35-41 \%$, in AML-M0.8,12

Patients with AML-M0 with RUNX1 mutation have been shown to have a higher male-to-female ratio and a higher median age at presentation. ${ }^{19} \mathrm{In}$ contrast, we did not observe significant differences in these parameters between $\mathrm{TdT}+$ and $\mathrm{TdT}-$ AML-M0 subgroup. RUNX1 mutations have been shown to be associated with CD34 and HLA-DR expression, and absence of CD33, CD15, CD19 and CD56 expression. ${ }^{19}$ We did not see any immunophenotypic differences between $\mathrm{TdT}+$ and TdT - AML-M0 in this study (Table 2). AML-M0 patients with RUNX1 mutation are reported to have a significantly higher serum LDH level compared with AML-M0 patients with wild-type RUNX1. ${ }^{19} \mathrm{In}$ our study, we did not find statistically significant differences in the serum LDH levels between patients with TdT + and TdT - AML-M0 (Table 1). RUNX1 mutation in AML is shown to be associated with poor induction response, failure to achieve CR, poor overall survival and poor disease-free survival. ${ }^{19}$ We did not see significant differences in these parameters between patients with $\mathrm{TdT}+$ and $\mathrm{TdT}-$ AML-M0 (Table 3). On the contrary, patients with TdT + AML-M0 showed better overall survival after receiving stem cell transplant as described above. However, we did identify one similarity. Trisomy 13 has been shown to be associated with RUNX1 mutation in AML-M0,8,9 and we also detected trisomy/tetrasomy of chromosome 13 only in TdT + AML-M0 cases $(3 / 11 ; 27 \%)$. Thus, despite the reported correlation between RUNX1 mutation and $\mathrm{TdT}$ expression, in this series the clinical presentation, immunophenotype and outcomes of patients with AML-M0 with TdT expression appear to differ from AML with RUNX1 mutation, except for a common association with trisomy/tetrasomy 13.

\section{Discussion}

This study had two major goals. First, we wished to assess the clinicopathological, immunophenotypic and cytogenetic features of AML-M0 as the definition of this disease has been refined in the current version of the WHO classification. Second, we wished to subdivide and assess AML-M0 cases according to TdT expression, based on recent data suggesting that TdT expression is a surrogate for RUNX1 gene mutations.

In the current WHO classification, the major change in criteria is the recognition of AML with myelodysplasia-related changes. Cases previously recognized as AML-M0, using either the FAB classification or the older 2001 WHO classification, are now reclassified as AML with myelodysplasia-related 
changes. Figure 1 shows an algorithmic approach for the subclassification of AML using the 2008 WHO classification. ${ }^{1}$ In this study, from a group of approximately 100 cases with FAB-M0 morphology as defined in the FAB system, we excluded 70 cases, now mostly reclassified as AML with myelodysplasiarelated changes. As a result, the AML-M0 category is now substantially smaller and our study represents a more accurate description of AML-M0 as currently defined. We are not aware of another study describing the clinicopathological and cytogenetic features of patients with AML-M0 using the current criteria.

Recently, gene expression profiling of AML-M0 cases has shown two distinct subgroups, one of which is fully associated with RUNX1 mutations ${ }^{10,12}$ and the other which shows poor overall survival. RUNX1 (also known as AML1 and by other names) is a member of the core binding factor (CBF) family of transcription factors. RUNX1 encodes the $\alpha$-subunit of CBF and is involved in the development of normal hematopoiesis. ${ }^{20}$ Most RUNX1 mutations are clustered in, but are not limited to, the Runt domain and result in defective DNA binding but active $\beta$-subunit binding. ${ }^{11}$ Chromosomal translocations involving the RUNX1 gene are well documented and have been associated with several types of leukemia such as the RUNX1-RUNX1T1 fusion transcript in AML with $\mathrm{t}(8 ; 21)(\mathrm{q} 22 ; \mathrm{q} 22){ }^{1}$ $R U N X 1$ can contribute to leukemogenesis, either as a tumor suppressor gene or an oncogene in various other hematological malignancies. ${ }^{21,22}$ Sporadic point mutations are frequently found in three leukemia entities: AML-M0, AML with MDS-related changes and secondary (therapy-related) MDS/AML. ${ }^{11,23,24}$ In AML-M0, half of the RUNX1 point mutations are reported to be biallelic, although the frequency varies with ethnicity. ${ }^{11}$

Comprehensive analysis for RUNX1 gene mutation requires analysis of eight exons and is not currently available in many molecular diagnostic laboratories including our own. However, the authors of the recent gene expression profiling study showed that TdT expression highly correlates with $R U N X 1$ mutation, and they proposed that TdT can be used as a surrogate for RUNX1 mutation status in AMLM0. As TdT expression is conveniently detected by flow cytometric analysis and is a standard of care for the workup of new acute leukemias, we subdivided the cases of AML-M0 into TdT $+(n=10)$ and TdT $-(n=20)$ groups.

In our study, we used a cutoff for TdT expression of $25 \%$ blasts, to avoid flow cytometry artifact of cell permeabilization and to ensure TdT expression by a significant number of blasts. Our results show that TdT expression breaks out AML-M0 cases into two distinctive groups. Patients with TdT + AML-M0 showed higher blood and bone marrow blast counts at the time of diagnosis, trisomy/tetrasomy 13 in a subset $(\sim 30 \%)$ of cases and complete absence of aberrations involving chromosomes 5 and 17. To our knowledge, no studies showing the features of TdT + AML-M0, as defined using the 2008 WHO classification, have been reported. A meta-analysis of features of TdT + cases within reported cohorts of AML-M0 cannot be performed because of inclusion of AML with myelodysplasia-related changes or therapy-related AML in the cohort, inclusion of cases showing higher expression of myeloperoxidase than allowed by the current diagnostic criteria and inconsistencies in immunophenotyping studies used in prior reports. ${ }^{25-28}$ Absence of chromosomal 5 abnormalities in our study is in contrast to a previous report showing abnormalities in chromosomes 5 and/or 7 in 5/19 (26\%) AML-M0 cases. ${ }^{28}$ Importantly, no correlation was observed between the TdT expression status and blast or bone marrow morphology in AMl-M0.

We observed a partial overlap, at best, between the features of patients with TdT + AML-M0 in this study and patients who have AML with RUNX1 mutations as reported by others. The frequency of TdT expression in AML-M0 in our study is comparable to the reported frequency of RUNX1 mutation in AML-M0. ${ }^{8,12}$ Patients with TdT + AML-M0 are predominantly male, as has been reported for patients with AML associated with RUNX1 mutations. ${ }^{19}$ All patients with gains of chromosome 13 had TdT + AML-M0. Association of trisomy 13 with RUNX1 mutation and increased FLT3 expression has been reported previously. ${ }^{8,9}$ However, unlike patients with AML associated with RUNX1 mutations, the TdT + AML-M0 group of patients in this study did not have a higher median age, higher serum LDH levels or a lower rate of CR upon induction chemotherapy and poor outcome as has been reported. Several explanations can be provided for partial but not complete overlap between the features of RUNX1-mutated AML and TdT + AML-M0. RUNX1 mutation (mono- versus bi-allelic, point mutation versus insertion/deletion, domain affected) may influence TdT expression, or TdT may be upregulated by pathways other than RUNX1 mutation.

The cytogenetic data in the $\mathrm{TdT}+$ and $\mathrm{TdT}-$ AML-M0 groups also suggests that these subsets are biologically different. All chromosome 13 aberrations in TdT + AML-M0 involved gain of chromosome 13, whereas chromosome 13 aberrations in TdT - AML-M0 involved losses. Similarly, aberrations involving chromosomes 5 and 17 were limited to the TdT - AML-M0. It is possible that in TdT + AML-M0 with trisomy 13, higher levels of FLT3 could contribute to a poorer outcome. Although we did not test for FLT3 mRNA expression levels, we did not notice any differences in FLT3 mutation status between $\mathrm{TdT}+$ and TdT - AML-M0. Interestingly, the gene expression signature in AML-M0 with RUNX1 mutation showed a unique signature of genes, many of which are related to early B-cell development. ${ }^{10}$ Our study did not show association of TdT expression with expression of lymphoid markers in AML-M0. 
TdT encodes a DNA polymerase normally expressed during early stages of pre-B and pre-T lymphocyte development. ${ }^{29}$ Some reports suggest that TdT expression in AML-M0 may reflect bi-phenotypic acute leukemia with myeloid predominance. In addition, frequent monoclonal IGH/TCRB rearrangements have been reported in AML-M0 with TdT expression. ${ }^{18}$ We detected monoclonal antigen receptor rearrangements in 6 of $10(60 \%) \mathrm{TdT}+$ AML-M0 cases, similar to a previously reported frequency of $61 \%(8 / 13)$ in TdT + AML-M0. ${ }^{18}$ No statistically significant difference was observed in the frequency of monoclonal gene rearrangements or expression of T-/B-cell markers between TdT + and TdT - AML-M0. This was true even when T-/B-cell marker expression was correlated to the amount of $\mathrm{TdT}$ expression. These findings suggest that TdT expression does not correlate with partial lymphoid differentiation in AML-M0 as defined using the 2008 WHO criteria. TdT expression in AML-M0 appears to correlate with the minimally differentiated nature of the blasts rather than a representation of a lymphoblastic component.

Our study is limited by not having RUNX1 mutation analysis for comparison with TdT expression; however, a goal of the study was to see if cumbersome RUNX1 analysis can be replaced with a more convenient assessment of TdT expression. From previously reported studies, it is evident that while there is a correlation between RUNX1 mutation and TdT expression as a whole in the study group, it may not be true for an individual patient. ${ }^{10,12}$ Also, by using the current WHO classification criteria for the group of AML-M0, the sample size has become smaller. Some of the properties of TdT + AML and TdT - AML-M0 that trended towards being different, but could not reach statistical significance, need to be investigated in a larger cohort of patients.

Importantly, significant improvements in overall survival were noted with stem cell transplant for patients with TdT + AML-M0 compared to TdT AML-M0. TdT + AML-M0 patients who received a stem cell transplant showed better outcome compared to TdT + AML-M0 patients who did not. No significant contributing factors could be identified that could explain these differences. The findings suggest that TdT expression may correlate with unique biological properties that directly or indirectly contribute to the beneficial effect of stem cell transplant. Owing to the retrospective nature of the study, the patients were not uniformly treated. The observation that TdT + AML-M0 patients show beneficial effect of stem cell transplant needs to be confirmed in a controlled clinical study with uniformly treated patients.

In summary, in this study we show that patients with TdT + AML-M0 show different clinical features than those reported for AML with RUNX1 mutation. However, TdT + AML-M0 shows clinicopathological features distinct from TdT - AML-M0 and TdT + AML-M0 patients seem to benefit from stem cell transplant. TdT expression can be con- veniently assessed using either flow cytometric analysis of bone marrow aspirate and peripheral blood or by immunohistochemistry staining of paraffin-embedded bone marrow biopsy and clot sections. Further clinical studies are required to confirm and elaborate the beneficial effects of TdT expression in the setting of stem cell transplant for patients with AML-M0.

\section{Disclosure/conflict of interest}

The authors declare no conflict of interest.

\section{References}

1 Arber D, Brunning R, Orazi A, et al. Acute myeloid leukemia, not otherwise specified. In: Swerdlow S, Campo E, Harris N, et al. (eds). WHO Classification of Tumours of Haematopoietic and Lymphoid Tissues. IARC Press: Lyon; 2008, pp 130-131.

2 Bennett JM, Catovsky D, Daniel MT, et al. Proposal for the recognition of minimally differentiated acute myeloid leukaemia (AML-MO). Br J Haematol 1991;78:325-329.

3 Amadori S, Venditti A, Del Poeta G, et al. Minimally differentiated acute myeloid leukemia (AML-M0): a distinct clinico-biologic entity with poor prognosis. Ann Hematol 1996;72:208-215.

4 Bene MC, Bernier M, Casasnovas RO, et al. Acute myeloid leukaemia M0: haematological, immunophenotypic and cytogenetic characteristics and their prognostic significance: an analysis in 241 patients. Br J Haematol 2001;113:737-745.

5 Stasi R, Del Poeta G, Venditti A, et al. Analysis of treatment failure in patients with minimally differentiated acute myeloid leukemia (AML-M0). Blood 1994;83:1619-1625.

6 van't Veer MB. The diagnosis of acute leukemia with undifferentiated or minimally differentiated blasts. Ann Hematol 1992;64:161-165.

7 Cuneo A, Ferrant A, Michaux JL, et al. Cytogenetic profile of minimally differentiated (FAB M0) acute myeloid leukemia: correlation with clinicobiologic findings. Blood 1995;85:3688-3694.

8 Dicker F, Haferlach C, Kern W, Haferlach T, Schnittger S. Trisomy 13 is strongly associated with AML1/ RUNX1 mutations and increased FLT3 expression in acute myeloid leukemia. Blood 2007;110:1308-1316.

9 Silva FP, Lind A, Brouwer-Mandema G, Valk PJ, Giphart-Gassler M. Trisomy 13 correlates with RUNX1 mutation and increased FLT3 expression in AML-M0 patients. Haematologica 2007;92:1123-1126.

10 Silva FP, Swagemakers SM, Erpelinck-Verschueren C, et al. Gene expression profiling of minimally differentiated acute myeloid leukemia: $\mathrm{MO}$ is a distinct entity subdivided by RUNX1 mutation status. Blood 2009;114:3001-3007.

11 Osato M. Point mutations in the RUNX1/AML1 gene: another actor in RUNX leukemia. Oncogene 2004;23: 4284-4296.

12 Silva FP, Almeida I, Morolli B, et al. Genome wide molecular analysis of minimally differentiated acute myeloid leukemia. Haematologica 2009;94:1546-1554. 
13 Yin CC, Medeiros LJ, Glassman AB, Lin P. $\mathrm{t}(8 ; 21)(\mathrm{q} 22 ; \mathrm{q} 22)$ in blast phase of chronic myelogenous leukemia. Am J Clin Pathol 2004;121:836-842.

14 Lin P, Jones D, Medeiros LJ, et al. Activating FLT3 mutations are detectable in chronic and blast phase of chronic myeloproliferative disorders other than chronic myeloid leukemia. Am J Clin Pathol 2006; 126:530-533.

15 Zuo Z, Chen SS, Chandra PK, et al. Application of COLD-PCR for improved detection of KRAS mutations in clinical samples. Mod Pathol 2009;22:1023-1031.

16 Ravandi F, O’Brien S, Jorgensen J, et al. Phase II study of cladribine followed by rituximab in patients with hairy cell leukemia. Blood 2011;118:3818-3823.

17 Vega F, Medeiros LJ, Jones D, et al. A novel four-color PCR assay to assess T-cell receptor gamma gene rearrangements in lymphoproliferative lesions. Am J Clin Pathol 2001;116:17-24.

18 Seremetis SV, Pelicci PG, Tabilio A, et al. High frequency of clonal immunoglobulin or T cell receptor gene rearrangements in acute myelogenous leukemia expressing terminal deoxyribonucleotidyltransferase. J Exp Med 1987;165:1703-1712.

19 Tang JL, Hou HA, Chen CY, et al. AML1/RUNX1 mutations in 470 adult patients with de novo acute myeloid leukemia: prognostic implication and interaction with other gene alterations. Blood 2009;114: 5352-5361.

20 Friedman AD. Cell cycle and developmental control of hematopoiesis by Runx1. J Cell Physiol 2009;219:520-524.

21 Look AT. Oncogenic transcription factors in the human acute leukemias. Science 1997;278:1059-1064.
22 Silva FP, Morolli B, Storlazzi CT, et al. Identification of RUNX1/AML1 as a classical tumor suppressor gene. Oncogene 2003;22:538-547.

23 Harada H, Harada Y, Niimi $\mathrm{H}$, et al. High incidence of somatic mutations in the AML1/RUNX1 gene in myelodysplastic syndrome and low blast percentage myeloid leukemia with myelodysplasia. Blood 2004; 103:2316-2324.

24 Harada Y, Harada H. Molecular pathways mediating MDS/AML with focus on AML1/RUNX1 point mutations. J Cell Physiol 2009;220:16-20.

25 Kaleem Z, White G. Diagnostic criteria for minimally differentiated acute myeloid leukemia (AML-M0). Evaluation and a proposal. Am J Clin Pathol 2001;115: 876-884.

26 Kotylo PK, Seo IS, Smith FO, et al. Flow cytometric immunophenotypic characterization of pediatric and adult minimally differentiated acute myeloid leukemia (AML-M0). Am J Clin Pathol 2000;113: 193-200.

27 Segeren CM, de Jong-Gerrits GC, van’t Veer MB. AMLMO: clinical entity or waste basket for immature blastic leukemias? A description of 14 patients. Dutch Slide Review Committee of Leukemias in Adults. Ann Hematol 1995;70:297-300.

28 Venditti A, Del Poeta G, Buccisano F, et al. Minimally differentiated acute myeloid leukemia (AML-M0): comparison of 25 cases with other French-AmericanBritish subtypes. Blood 1997;89:621-629.

29 Silverstone AE, Cantor H, Goldstein G, Baltimore D. Terminal deoxynucleotidyl transferase is found in prothymocytes. J Exp Med 1976;144:543-548. 